\title{
COMPARISON OF NON-INVASIVE METHODS FOR THE DETECTION OF CORONARY ATHEROSCLEROSIS
}

\author{
Angela Bacelar Albuquerque Bampi, ${ }^{I}$ Carlos Eduardo Rochitte, ${ }^{I I}$ Desiderio

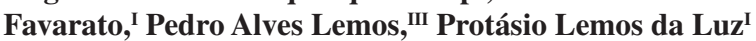

doi: $10.1590 / \mathrm{S} 1807-59322009000700012$

Bampi ABA, Rochitte CE, Favarato D, Lemos PA, da Luz PL. Comparison of non-invasive methods for the detection of coronary atherosclerosis. Clinics. 2009;64(7):675-82.

BACKGROUND: Non-invasive detection of atherosclerosis is critical for its prevention.

Objective: To correlate non-invasively detectable indicators of coronary atherosclerosis, or Coronary Artery Disease (i.e., classical risk factors, hs-CRP test results, carotid intima-media thickness, endothelial function, ankle-brachial index and calcium score by computed tomography) with the extent of coronary disease assessed by the Friesinger index from conventional coronary angiography. METHODS: We conducted a prospective study of 100 consecutive patients, mean age $55.1 \pm 10.7$ years, $55 \%$ men and $45 \%$ women. Patients with acute coronary syndrome, renal dialytic insufficiency, collagen disease and cancer were not included. All patients were subjected to clinical evaluation and laboratory tests. Endothelial function of the brachial artery and carotid artery were evaluated by high-resolution ultrasound; ankle-brachial index and computed tomography for coronary determination of calcium score were also performed, and non-HDL cholesterol and TG/HDL-c ratio were calculated. All patients were subjected to coronary angiography at the request of the assistant physician. We considered patients without an obstructive lesion $(<29 \%$ stenosis $)$ demonstrated by coronary angiography to be normal.

RESULTS: Univariate analysis showed that calcium score, HDL-c, TG/HDL ratio and IMT were significantly correlated with the Friesinger index. However, multivariate analysis indicated that only calcium score and low HDL-c levels correlated significantly with the extension of CAD. On the other hand, hs-CRP, LDL-c, flow-mediated dilation, and Framingham score did not correlate with the Friesinger index. ROC analysis showed that calcium score, HDL-c and TG-HDL ratio accurately predicted extensive CAD in a statistically significant manner.

CONCLUSION: It is possible to approximately determine the presence and extent of CAD by non-invasive methods, especially by calcium score, HDL-c and TG/HDL-c ratio assays.

KEYWORDS: Risk factors; Lipids; C-reactive protein; Tomography; Atherosclerosis

\section{INTRODUCTION}

Atherosclerosis is an inflammatory disease that affects all arteries and can lead to ischemia of the heart, brain or

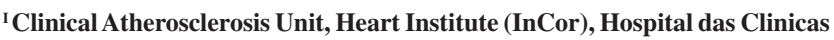
da Faculdade de Medicina da Universidade de São Paulo - São Paulo/SP, Brazil. II Radiology Unit, Heart Institute (InCor), Hospital das Clinicas da Faculdade de Medicina da Universidade de São Paulo - São Paulo/SP, Brazil.

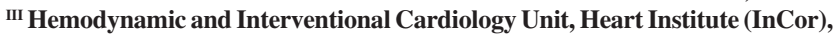
Hospital das Clinicas da Faculdade de Medicina da Universidade de São Paulo - São Paulo/SP, Brazil. Email: daluzp@incor.usp.br

Tel: 55113069.5447

Received for publicaiton on February 08, 2009

Accepted for publication on April 26, 2009
}

extremities. ${ }^{1}$ It is the result of complex interactions between genetic and environmental factors which induce the arterial wall to respond to stimuli through the action of endothelial cells, smooth muscle, inflammatory cells and platelets, ${ }^{1}$ leading to formation of plaques. The initial stages occur among children and young people and are silent and progress slowly; clinical manifestations generally appear in middle age. $^{2}$ However, the first event triggered by atherosclerosis can be fatal. Recently, there has been an increase in recognition of the importance of subclinical atherosclerosis. For instance, elderly people with subclinical disease have a worse prognosis than those without this disease early disease. ${ }^{3}$ Furthermore, progression of atherosclerosis can 
be significantly reduced when dyslipidemia is treated by statins; ${ }^{4}$ reduction in cardiovascular events after statin treatment has also been widely documented. ${ }^{5}$

Therefore, non-invasive documentation of atherosclerotic lesions has become an important objective for early treatment as well as preventive measures. Many strategies have been proposed to this end, but a thorough comparison of these approaches is still incomplete. Here, we sought to compare clinical indicators, laboratory tests and direct arterial vessel indices with standard coronary angiography to determine their ability to predict the extent of CAD.

\section{METHODS}

One hundred consecutively admitted patients of both sexes who underwent coronary angiography for diagnostic purposes between January 2005 and July 2007 were enrolled in our prospective study. All patients signed a form indicating their informed consent and underwent a clinical exam. Measurements of total cholesterol, HDL-c, LDL-c, triglycerides, fasting plasma glucose and hsCRP were also taken for each patient. Non-invasive tests included endothelial function studies, carotid ultrasound, calcium scoring by multislice coronary tomography and measurement of the ankle-brachial index. Exclusion criteria were acute coronary syndromes, atrial fibrillation, cancer, obstructive pulmonary illness, dialytic renal insufficiency or collagen illnesses. The research protocol was approved by the Ethics Committee of our institution.

Clinical evaluation: Subjects were categorized as smokers if they reported smoking any number of cigarettes within the six months before the study; otherwise they were labeled as non-smokers. Subjects were defined as having hypertension if they displayed a sitting systolic blood pressure $\geq 130 \mathrm{mmHg}$, diastolic blood pressure of $>85$ $\mathrm{mmHg}$, or reported use of pressure-lowering medication. Diabetes mellitus was considered present if the subject reported current use of oral hypoglycemiant or insulin, or presented with fasting glucose levels above $126 \mathrm{mg} / \mathrm{dL}$. Family history of hypertension was defined as a history of coronary disease in first first-degree relatives occurring before 55 years of age in men and 60 years of age in women. Weight, height and waist circumference were taken, and BMI was calculated for all subjects. Endothelial function, carotid intima-media thickness and ankle-brachial index study were assessed as described below.

Endothelial function was evaluated by flow-mediated dilation (FMD) in the left brachial artery. The left brachial artery was scanned with an ultrasound probe over a longitudinal section 3 to $5 \mathrm{~cm}$ above the right elbow. A pneumatic tourniquet was placed around the forearm distal to the target artery, then inflated to $250 \mathrm{mmHg}$ and maintained for $5 \mathrm{~min}$. Vessel recovery was assessed by a further resting scan 15 min later. Sublingual nitrate (5 $\mathrm{mg}$ ) was administered, and a final scan was performed 5 min later. The diameter of the brachial artery was measured from the anterior to the posterior interface between the media and adventitia at a fixed distance. The mean of end-diastole diameter was calculated from five cardiac cycles synchronized with the R-wave peaks on the electrocardiogram. The change in diameter was expressed as the percent change relative to that at the initial resting scan. ${ }^{6}$ The pulse wave velocity profile of blood flow was simultaneously recorded. All measurements were performed between 8:00 and 11:00 AM in a temperature-controlled room with the subjects in a resting, supine state. Subjects abstained from alcohol, caffeine, tobacco and food for 12 $\mathrm{h}$ before the study. Long-acting vasoactive medications, including calcium channel blockers, $B$-adrenergic blocking agents, nitrates and converting enzyme inhibitors were discontinued for $12 \mathrm{~h}$ before the study. A 7.5 MHz linear array ultrasound probe attached to an ultrasound system (ATL-APOGEE 800 Plus $^{\mathrm{TM}}$ ) was used.

The normal coefficients of variation in our laboratory were $5.84 \pm 0.25 \%$ for measurements of FMD and $3.97 \pm 0.24 \%$ for measurements of NTG. ${ }^{7,8}$ The coefficient of variation for the reproducibility of the ultrasound was $9.77 \pm 0.82 \%$ for determination of FMD and $7.24 \pm 0.49 \%$ for determination of NTG. ${ }^{7,8}$

\section{Carotid B-mode ultrasonography and intima-media thickness assessment}

Intima-media thickness of the bilateral common right and left carotid arteries was measured at $1 \mathrm{~cm}$ below the carotid artery bifurcationwith an ATL (APOGEE 800 Plus $^{\mathrm{TM}}$ ) linear array probe $(7.5 \mathrm{MHz}) .{ }^{9}$ Each common carotid artery was evaluated with the subject in the supine position and head turned slightly to the contralateral side. Images were gated to ECG, and seven acquired images were selected: one for calibration, three in the first $6 \mathrm{~ms}$ of the $\mathrm{T}$ wave (from three different beats) to determine the largest diameter, and three at peak of the $\mathrm{R}$ wave to determine the smallest diameter. All exams were videotaped with a VHS system. The measurements were performed with the aid of software specifically designed for analyses of diameter, arterial flow and intima-media thickness developed at The Heart Institute.

All images were interpreted by a technician who was specifically trained in the assessment of IMT. Images were also visually inspected for plaques. The thickest value was chosen for represent the IMT of each patient. Plaques were considered present if any IMT value was over $1.0 \mathrm{~mm}$. 
Ankle-brachial index is the ratio of the systolic blood pressure (SBP) at the ankle to the SBP in the arm. It is assumed that the decrease of effective perfusion pressure distal to the stenosis is roughly proportional to the severity of the occlusive disease. A cuff size appropriate for arm size was used for the brachial and ankle SBP. A hand-held Doppler was used to assess bilateral brachial, tibial and dorsalis pedis arteries. At each ankle (right and left), the higher of the two pressures measured in each leg was used as the numerator for same-side ABI calculation. ${ }^{10,11}$

Laboratory measurements Blood samples for lipid measurements were drawn after the subject had fasted for $12 \mathrm{~h}$. Total cholesterol and triglyceride serum levels were measured with enzymatic methods. High-density lipoprotein levels was determined after precipitation of apolipoprotein B. Low-density lipoprotein cholesterol concentrations were computed using the Friedewald formula for triglyceride levels $\leq 350 \mathrm{mg} / \mathrm{dL}$. Non-HDL cholesterol and $\mathrm{Tg} / \mathrm{HDL}$ ratio were also calculated. High-sensitivity C-Reactive Protein (hs-CRP) levels were measured by latex immunonephelometry using Dade Behring ${ }^{\mathrm{TM}}$ reagents. Glucose levels were measured by colorimetric enzymatic methods using hexoquinase.

The Toshiba Multi-Slice Aquilion $64^{\mathrm{TM}}$ system was used to perform 64-Slice Multislice Computed Tomography. Images were obtained using a 64-slice ( $3 \mathrm{~mm}$ thickness) protocol with image acquisition triggered to $80 \%$ of the electrocardiographic RR interval while respiration was held. Calcium scores were calculated according to Agatston's method $^{12,13}$ using a workstation Vitrea TM2 ${ }^{\mathrm{TM}}$ with software specific to and validated for the assessment. Trained scan readers were blinded to the study parameters. A focus of coronary calcium was defined as the presence of four or more contiguous pixels measuring $>130$ Hounsfield units. Total coronary calcium scores (CCS) were determined from the sum of individual scores of the four major epicardial coronary arteries. A scan was considered positive for CAD when the total CCS was $>0$.

Coronary Angiography: All patients had undergone coronary angiography within the previous six months. The time elapsed between angiography and the non-invasive measurements ranged from one week to six months; however, $98 \%$ were within four months. Most exams (95\%) were performed in our hemodynamic laboratory, using Phillips systems (Intergris BH 3000) in the typical orthogonal projections and a percutaneous technique with arterial access by brachial or femoral arteries. The analyses were performed by a physician blinded to the study. We used the Friesinger index ${ }^{14}$ for assessment of the extent of coronary disease. The scores in this index range from 0 to 15 . Each of the three main coronary arteries is scored separately from zero to five. The scores are: 0: no arteriographic abnormalities; 1: trivial luminal narrowing of $<29 \%$; 2: localized luminal narrowing of $30-68 \%$; 3 : multiple sites of luminal narrowing at 30-68\%; 4: luminal narrowing of $69-100 \%$ without $100 \%$ occlusion of proximal segments; 5: complete $(100 \%)$ obstruction of a proximal segment.

Statistics: The sample size was calculated assuming $40 \%$ simultaneous occurrence of abnormal intima-media thickness and coronary disease. This indicated that analysis of a minimum of 80 patients was necessary to show a statistically significant association between these two variables; we included 100 patients to ensure a safe margin in the event of losses. Continuous variables were described by mean and standard deviation, and frequency distributions were used to describe non-continuous variables. Chi-square and non-parametric ANOVA (Kuskal-Wallis) analyses were used to assess association. Multivariate analysis was performed by logistic regression.

\section{RESULTS}

Clinical characteristics: The clinical characteristic of the patients are summarized in Table 1. Of our 100 subjects, 63 patients presented with obstructive $\mathrm{CAD}$, and 37 showed no coronary lesions. The mean age was $55.1 \pm 10.7$ years; $55 \%$ of subjects were men, and $45 \%$ were women. Arterial hypertension was present in $75 \%$, dyslipidemia in $47 \%$ and diabetes mellitus in $28 \%$. Smokers made up $15 \%$ of the sample.

Table 2 shows the results of laboratory tests. Thirtyeight patients $(38 \%)$ had LDC-c $\geq 130 \mathrm{mg} / \mathrm{dL}$. These measurements varied somewhat by gender; 36 of 45 women (80\%) had HDL-c $<50 \mathrm{mg} / \mathrm{dL}$, while only 24 of 55 (43.6\%) men had values $<40 \mathrm{mg} / \mathrm{dL}$. Hs-CRP values were over 5 $\mathrm{mg} / \mathrm{L}$ in $16(18.3 \%)$ subjects. Framingham scores indicated low risk for $17.4 \%$ of subjects, intermediate risk for $48.8 \%$ and high risk for $33.8 \%$. Triglyceride values were $>150 \mathrm{mg} /$ $\mathrm{dL}$ in 44 patients $(44 \%)$.

Table 3 shows the results of non-invasive indices. Flowmediated dilation (FMD) was above $8 \%$ in 11 men (13\%) and above $10 \%$ in six women $(7.1 \%)$. IMT values were below $1.0 \mathrm{~mm}$ in 58 patients $(67.4 \%)$ and above $1.0 \mathrm{~mm}$ in 28 patients $(32.5 \%)$. The TG/HDL ratio was $<3.75$ in 53 patients (53\%) and $>3.75$ in 47 patients (47\%).

Patients were given appropriate medications to control coronary disease as shown in Table 1 . 
Table 1- Clinical characteristics of patients

\begin{tabular}{|c|c|c|}
\hline Characteristic & $\mathrm{N}$ & $\%$ \\
\hline Patients & 100 & 100 \\
\hline Age (years) & $55.1 \pm 10.7$ & \\
\hline Men & 55 & 55 \\
\hline \multicolumn{3}{|l|}{$\begin{array}{l}\text { Coronary Angiography (Friesinger } \\
\text { index) }\end{array}$} \\
\hline 0 & 37 & 37 \\
\hline $1-4$ & 7 & 7 \\
\hline $5-9$ & 32 & 32 \\
\hline $10-15$ & 24 & 24 \\
\hline HAS & 75 & 75 \\
\hline $\mathrm{DM}$ & 28 & 28 \\
\hline Dyslipidemia & 36 & 36 \\
\hline Smoking & 15 & 15 \\
\hline Previous AMI & 30 & 30 \\
\hline Waist circumference $(\mathrm{cm})$ & $97.4 \pm 9.9$ & \\
\hline BMI $\left(\mathrm{kg} / \mathrm{m}^{2}\right)$ & $32.1 \pm 36.5$ & \\
\hline \multicolumn{3}{|l|}{ Medications } \\
\hline Aspirin & 90 & 90 \\
\hline Statin & 70 & 70 \\
\hline Beta-blockers & 62 & 62 \\
\hline Angiotensin-converting enzyme inhibitor & 47 & 47 \\
\hline Nitrate & 30 & 30 \\
\hline Calcium channel blockers & 20 & 20 \\
\hline Diuretics & 20 & 20 \\
\hline Oral hypoglicemiants/Insulin & 20 & 20 \\
\hline Angiotensin I receptor block & 6 & 6 \\
\hline Fibrates & 4 & 4 \\
\hline
\end{tabular}

Table 2- Laboratory test scores

\begin{tabular}{lcc}
\hline & $\mathrm{N}$ & Mean $\pm \mathrm{SD}$ \\
\hline $\begin{array}{l}\text { Cholesterol Total }(\mathrm{mg} / \\
\mathrm{dL})\end{array}$ & 100 & $200.5 \pm 53.8$ \\
$\begin{array}{l}\text { Non-HDL-c cholesterol } \\
(\mathrm{mg} / \mathrm{dL})\end{array}$ & 100 & $154 \pm 47.5$ \\
HDL-c $(\mathrm{mg} / \mathrm{dL})$ & 100 & $45.9 \pm 5.5$ \\
LDL-c (mg/dL) & 100 & $122.8 \pm 51.5$ \\
Triglycerides (mg/dL) & 100 & $203.0 \pm 339.3$ \\
TG/HDLc & 100 & $5.5 \pm 9.2$ \\
hs-CRP (mg/L) & 86 & $4.9 \pm 12.4$ \\
Glucose (mg/dL) & 100 & $114.5 \pm 51.0$ \\
\hline
\end{tabular}

Abbreviations: hs-CRP: high sensitivity c-reactive protein, TG: triglycerides
Table 3- Non-invasive test results (mean \pm SD)

\begin{tabular}{llc}
\hline & $\mathrm{N}$ & Mean \pm SD \\
\hline FMD $(\%)$ & 84 & $4.8 \pm 5.7$ \\
FID $(\%)$ & 84 & $16.0 \pm 8.07$ \\
IMT $(\mathrm{mm})$ & 90 & $0.77 \pm 0.22$ \\
ABI & 89 & $1.05 \pm 0.7$ \\
Agatston score & 95 & $529.5 \pm 930 . .9$ \\
\hline
\end{tabular}

Abbreviations: FMD: flow-mediated dilation, FID: flow-independent dilation, IMT: intima-media thickness, ABI: ankle-brachial index.

\section{Exploratory statistic tests}

There was a weak positive correlation between Framingham score and age $\left(\mathrm{R}^{2}=0.194\right)$. When Framingham score and IMT (dichotomized at $1 \mathrm{~mm}$ ) were cross-tabulated, a borderline positive association was observed $(\mathrm{p}=0.053)$. Univariate analysis indicated a positive association ( $\mathrm{p}=0.004)$ between extensive coronary atherosclerosis defined by Friesinger index $\geq 5$ and abnormal IMT.

The univariate analyses of the linear to linear association between extensive coronary disease (Friesinger index $\geq 5$ ) and quartiles of calcium score and lipid profile presented the following results: quartiles of calcium score, $p<0.0001$; quartiles of HDL, $\mathrm{p}=0.0001$; quartiles of TG/HDL-c, $\mathrm{p}=0.002$; quartiles of total cholesterol, $\mathrm{p}=0.007$ (this correlation was inconsistent due to the negative correlation between total cholesterol quartiles and the presence of extensive coronary disease, i.e., there were fewer patients with extensive coronary disease in the third and fourth cholesterol quartiles); quartiles of LDL, $\mathrm{p}=0.06$; quartiles of triglycerides, $p=0.414$. When the strength of the same variables for exclusion or indication of coronary lesions was tested, the results were as follows: calcium score, $\mathrm{p}=0.0001$; HDL-c, $\mathrm{p}=0.003$; TG/HDL-c, $\mathrm{p}=0.013$; LDLc, $\mathrm{p}=0.293$; total cholesterol, $\mathrm{p}=0.167$; triglycerides, $\mathrm{p}=0.695$. Thus, calcium score was the best discriminator for the extent and presence of coronary lesions, followed by HDL and TG/HDL ratio. These results are shown in Table 4.

Association strengths were tested by comparing the fourth and first quartiles, which showed the following statistically significant results: coronary calcium score OR 585, 95\%CI (26.8-12784), $\mathrm{p}<0.0001$; HDL-c OR $0.11,95 \%$ CI $(0.03-0.39), \mathrm{p}=0.0007$; TG/HDL-c OR $5,5,95 \mathrm{CI}(1,6-18,4), \mathrm{p}=0.01$. No significant statistical associations were found with other lipid variables.

The best correlation index was found between the Friesinger index and Agatston calcium score. Thus, patients with a zero calcium score had very low frequency of obstructive disease, and the Friesinger index increased with increasing calcium score (Fig. 1). HDL-c also exhibited 
Table 4- Frequency of extensive coronary disease (Friesinger index $\geq 5$ ) by quartiles of coronary calcium score and lipid variables

\begin{tabular}{|c|c|c|c|c|c|c|c|c|c|c|c|c|}
\hline & \multicolumn{2}{|c|}{$\mathrm{CCS}$} & \multicolumn{2}{|c|}{$\mathrm{TC}$} & \multicolumn{2}{|c|}{ LDL } & \multicolumn{2}{|c|}{ HDL } & \multicolumn{2}{|c|}{ TG } & \multicolumn{2}{|c|}{ TG/HDL } \\
\hline \multicolumn{13}{|c|}{ Friesinger Index frequency $(\%)$} \\
\hline & 0 & $\geq 5$ & 0 & $\geq 5$ & 0 & $\geq 5$ & 0 & $\geq 5$ & 0 & $\geq 5$ & 0 & $\geq 5$ \\
\hline Q1 & 84.4 & 6.3 & 24 & 76 & 24 & 72 & 26.9 & 73.1 & 48 & 44 & 56 & 32 \\
\hline Q2 & 41.2 & 47.1 & 40 & 56 & 46.2 & 50 & 20.8 & 72 & 23.1 & 65.4 & 38 & 53.8 \\
\hline Q3 & 8.3 & 83.3 & 40 & 56 & 34.6 & 61.5 & 37.5 & 54.2 & 41.7 & 54.3 & 28 & 68 \\
\hline Q4 & 0 & 100 & 44 & 36 & 43.5 & 39.1 & 64 & 24 & 36 & 60 & 25 & 70.2 \\
\hline
\end{tabular}

$\mathrm{Q}=$ quartile

a significant negative relationship with Friesinger index $(\mathrm{p}=0.0001)$. Note that the Friesinger index is zero with HDL-c $>50 \mathrm{mg} / \mathrm{dL}$ from median (Figure 1). A positive association was also observed between the Friesinger index and TG/HDLc ratio. Each increment of the ratio was accompanied by an increase of the extent of coronary disease
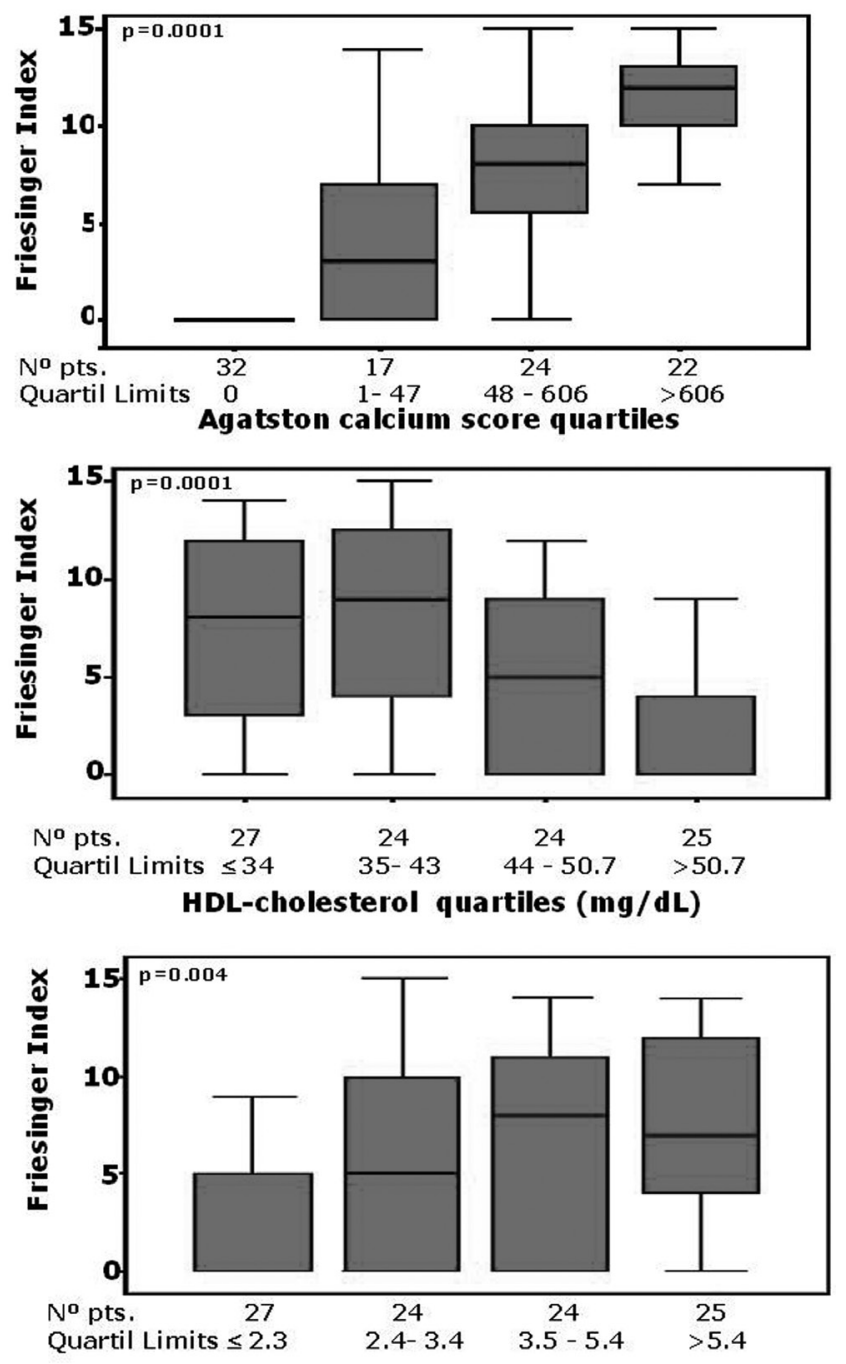

Triglycerides/HDL-cholesterol quartiles

Figure 1 - Friesinger index boxplot distribution by Agatston quartiles, HDL-c quartiles and TG/HDL-c quartiles $(\mathrm{p}=0.004)$ (Fig. 1). There was $28 \%$ discordance between TG/HDL-C and HDL-C, e.g., nine of 61 (14.8\%) patients presented a normal TG/HDL-C ratio and low HDL-C, and 19 of $39(88.7 \%)$ patients exhibited abnormally elevated TG/ HDL-C ratio but normal HDL-C levels. This discrepancy was found in 14 of 56 (25\%) patients with extensive coronary disease and in 19 of $49(31.8 \%)$ of those with less extensive disease.

Other variables, such as age, smoking, Framingham score, BMI, diabetes mellitus, total cholesterol, LDL-c, non HDL-c cholesterol, triglycerides, hs-CRP, FMD, waist circumference and ABI, were not significantly associated with extent of coronary disease as determined by Friesinger index.

Multivariate analyses (logistic regression) associated the following variables with the Friesinger index: calcium score, $\mathrm{R}=6.6,95 \%$ CI: 3.16-13.72, $\mathrm{p}<0.0001$; HDL-c, $\mathrm{OR}=0.30$, 95\% CI: 0.18-0.51, $\mathrm{p}<0.0001$. A significant association was also observed for TG/HDL ratio, $\mathrm{OR}=0.7$, 95\% CI: 0.48 $1.05, \mathrm{p}=0.085$.

\section{Analysis by the ROC curve}

The variables for which association remained statistically significant in multivariate analysis (i.e., calcium score, HDL-c and TG/HDL-c ratio) were analyzed by means of ROC curves to determine accuracy in detecting extensive coronary disease (Friesinger index $\geq 5$ ). The variable with the greatest area under the curve was calcium score $(92.7 \%)$, followed by HDL-c (71.1\%) and TG/HDL-c ratio $(68 \%)$. The cut-off point of the Agatston calcium score, which has a better profile of accuracy, was 55 that showed $81 \%$ sensitivity and $90 \%$ specificity Simulations at different cut-off points of Agatston calcium score, TG/HDL-C ratio and HDL-C showed the following coordinates of sensitivity and specificity: Agatston equal to zero: sensitivity $70 \%$, specificity 96\%; Agatston equal to 100: sensitivity 95\%, specificity $60 \%$; TG/HDL-c (3.75): sensitivity $61.5 \%$, specificity 63\%; and HDL-C $40 \mathrm{mg} / \mathrm{dL}$ : sensitivity $70 \%$, specificity $65 \%$. 
When ROC curves were constructed using quartiles of these variables, the following ideal points emerged: calcium quartile 2.5 , corresponding to $90 \%$ sensitivity and $80 \%$ specificity; TG/HDL-c ratio quartile 1.5 with $70 \%$ sensitivity and $60 \%$ specificity; HDL-C quartile 1.5 with $75 \%$ sensitivity and $65 \%$ specificity. ROC curves are shown in Figure 2.

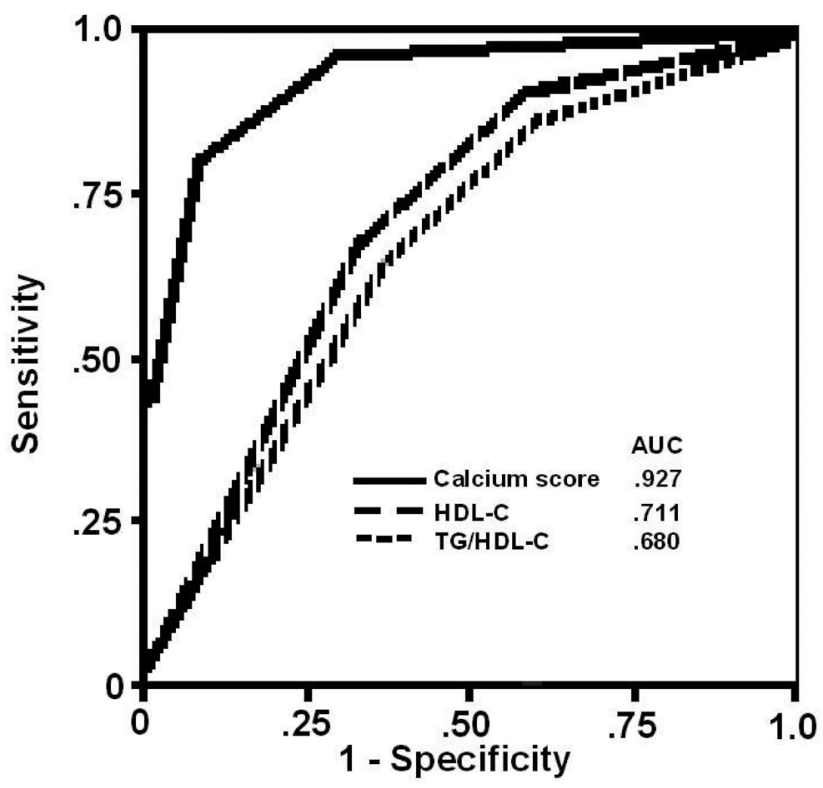

Figure 2 - ROC curves by quartiles of Agatston, TG/HDL-c and HDL-c for patients with a Friesinger index $\geq 5$

Since the calcium score was found to be the most sensitive and specific index for CAD detection, we also examined correlates of this variable. Agatston quartiles correlated positively with age $(\mathrm{p}<0.001)$ and IMT $(\mathrm{p}=0.002)$, however there was only a weak correlation with Framingham score $(p=0.079)$. We did not find significant correlation between Agatston score and triglyceride level, HDL-c, noncholesterol HDL-c, hs-CRP, waist circumference, FMD, ABI, BMI, HAS, DM or Framingham score.

\section{DISCUSSION}

The main findings of this study were that calcium score, HDL-C levels and TG/HDL-C ratio are the noninvasive parameters most strongly associated with the extent of coronary disease as assessed by Friesinger index from conventional coronary angiography. Several aspects of this study deserve discussion. Our population is a sample of the so-called "real world" of CAD in Brazil. We enrolled patients who represented the entire spectrum of CAD; our subjects included patients with no CAD as well as individuals with extensive CAD. It should be noted, however, that a normal luminogram does not necessarily guarantee that those arteries are free of atherosclerosis.

All patients enrolled in the study had some risk factors for CAD; $70 \%$ of them were already using lipid-lowering drugs in accordance with the guidelines for secondary prevention (64\% of our population had proven CAD). The most prevalent risk factor was hypertension, followed by dyslipidemia and diabetes. Our risk factor prevalence were similar to that found by Escoteguy et al that studied another Brazilian sample except by our higher prevalence of hypertension ${ }^{15}$. Smoking was unrelated to the presence of obstructive CAD. The prevalence of smoking was lower than indicated in data from national surveys, that showed variation from $21 \%$ to $36 \%{ }^{16,17}$. This lower incidence may be due to secondary prevention in patients already being treated for coronary disease

Framingham scores had no significant correlation with extent of CAD. However it might be antecipated because we studied a high risk population with indication of coronary angiography, e.g. we have worked with an homogenous population with a high prevalence of disease On the ohter hand Framingham score was designed to predict clinical coronary disease events but is not intended to correlate with coronary disease extension.

Our patients had slightly increased levels of triglycerides and glucose, according to Brazilian guidelines for dyslipidemia and diabetes. Total cholesterol, HDL-c and LDL-c, were within normal limits, probably due to the use of statins in the majority of patients. However, $38 \%$ had LDL-C $\geq 130 \mathrm{mg} / \mathrm{dL}$, which is considered high for the purposes of secondary prevention. Several studies have shown a correlation between CAD and those risk factors. ${ }^{18,19}$ In the Framingham study, ${ }^{18}$ the risk of CAD is 4.9 times higher in diabetic women and 2.1 times higher in men with diabetes. Low HDL-C is also among the risk factors for the Framingham score, while high HDL-C protects against CAD. ${ }^{18}$ HDL-C levels were satisfactory and the analysis of HDL-C quartiles showed an inverse relationship with the extent of CAD. Thus lower levels of HDL-C coincide with a higher Friesinger index, in agreement with the Lipid Research Center ${ }^{20}$ and Framingham studies, ${ }^{18}$ and a low HDL-C level is an independent risk factor for CAD. In Brazil, Magalhaes ${ }^{21}$ evaluated 165 patients that had undergone coronary artery bypass grafting and showed that, of the factors they studied, HDL-C below $35 \mathrm{mg} /$ $\mathrm{dL}$ was the only independent predictor of mortality at five years. Although several studies have identified hs-CRP as a sensitive marker for coronary events, ${ }^{22}$ we did not find a correlation between hs-RCP and Friesinger index or calcium score. The observed low correlation in the present study may also be related to the use of statins among patients in our study group, as statins have anti-inflammatory properties. ${ }^{23}$ 
The TG/HDL ratio correlated with Friesinger index but not with calcium score. We have no clear explanation for this paradox; increased TG/HDL ratio coincides with increased numbers of small and dense LDL particles that are more atherogenic, and thus subclinical atherosclerosis should be correlated with an abnormal ratio. However, ROC curves show that this relationship is of intermediate value. In a previous study of 374 patients, we also observed a significant correlation of elevated TG/HDL-C ratio with extensive CAD. ${ }^{24}$ We have also reported that high levels of TG/HDL-C ratio were associated with precocious manifestation of the disease in another 495 patients with $\mathrm{CAD}$, demonstrating the clinical utility of this ratio. ${ }^{25}$

Several correlations were tested between the Friesinger index and methods for the direct observation of coronary and peripheral arteries. Agatston score showed a positive correlation with the extent of coronary disease measured by the Friesinger index. These findings are consistent with studies that showed a higher incidence of coronary events in the presence of calcification of the coronary arteries. ${ }^{26}$ Several studies also showed calcium score as an useful tool for risk stratification. ${ }^{27}$ Agatston score increased in proportion with increasing age; the same direct correlation was observed in a Brazilian study that showed a positive correlation between age and coronary calcium score $(\mathrm{r}=$ $0.4, \mathrm{p}<0.01)^{28}$

Framingham score was weakly correlated with calcium score. A metanalysis carried out in asymptomatic subjects showed that an above-average calcium score was associated with acute myocardial infarction and death..$^{29}$ The predictive value of calcium score in patients at high risk is still unclear, but it is superior to that of traditional indices. The association of calcium score with Framingham score was assessed by Greenland et al., ${ }^{30}$ who followed 1312 asymptomatic, non-diabetic, middle age subjects 45 years or older for 8.5 years and found that a calcium score over 300 , as measured by coronary CT, was predictive of AMI or death from coronary artery disease $(\mathrm{p}<0.001)$, compared with a score of zero. Moreover, in subjects with Framingham scores higher than $10 \%$, an increased calcium score indicates a greater likelihood of coronary events, while in individuals with Framingham scores of $<10 \%$ this correlation did not occur. Therefore, in our study, calcium score emerged as the most important marker for both the presence and absence of CAD.

Measures of IMT showed a mean value of 0.77 $\mathrm{mm}$, which we defined as normal. Subjects with carotid thickening (IMT $\geq 1.0 \mathrm{~mm}$ ) exhibited a greater extent of coronary artery disease and a higher calcium score. These data confirm previous studies that showed a correlation between carotid intima-media thickness and increased cardiovascular event rates. ${ }^{31}$ For example, in the Kuopio Ischemic Heart Disease Risk Factor Study, the risk of acute myocardial infarction was shown to increase $11 \%$ for each increase of $0.1 \mathrm{~mm}$ in carotid IMT. ${ }^{32}$

Neither arterial endothelium-dependent nor -independent dilation were correlated with Friesinger index or with calcium score. This finding contrasts with those of previous works ${ }^{33}$ and can not be easily explained; however, the use of statins among our subjects is a plausible explanation. The ankle-brachial index was abnormal in only 13 patients; seven had values $\leq 0.9$, and six had values $\geq 1.3$. No correlation between ankle-brachial index and Friesinger index or calcium score was observed. Although several studies show abnormal ABI to be a marker of peripheral arterial disease, and its presence to be associated with coronary events, we could not confirm these results here.

In summary, we showed that a consistent estimate of the presence, extension or absence of coronary lesions by non-invasive methods is possible even in a cohort of realworld patients. Of course, there is no absolute precision in these findings, only approximation. However, these results suggest that certain patients should undergo more thorough investigations, while others should not; our findings might also inform guidelines for preventive measures.

\section{Limitations}

All patients included in the project had multiple risk factors for CAD or were known to have CAD. Thus, this population does not include truly normal individuals. Furthermore, many of the study subjects were taking medications; $70 \%$ were on statins. Thus, this is a select population; this fact could influence the outcome of some tests such as those for endothelial function, the hs-RCP and blood lipids. However, it is presently almost impossible to find CAD patients who are not taking any medications.

The mean age of the study group was 55 years, which is relatively low for the early detection of peripheral arterial disease. This may in part explain the absence of correlation between ABI and CAD. Despite these limitations, the study offers an objective, broad comparison of non-invasive tests for $\mathrm{CAD}$ detection and quantification.

\section{CONCLUSIONS}

Non-invasive methods can predict the presence and extent of coronary artery disease. Coronary calcium score was the most accurate predictor, followed by HDL-c levels and the triglyceride:HDL-c ratio. 


\section{REFERENCES}

1. Ross R. Atherosclerosis: an inflammatory disease. N Engl J Med. 1999;340:115-26.

2. Pathobiological Determinants of Atherosclerosis in Youth (PDAY) Research Group. Relationship of atherosclerosis in young men to serum lipoprotein cholesterol concentration and smoking. A preliminary report from the PDAY. J Am Med Ass.1990;264:3018-24.

3. Kuller L, Borthani N, Furberg C, Gardin J, Manolio T, O'Leary D, et al. Prevalence of Subclinical Atherosclerosis and Cardiovascular Disease and Association with Risk Factors in the Cardiovascular Health Study. Am J Epidemiol.1994;139:1164-79.

4. Crouse III JR, Raichlen JS, Riley WA, Evans GW, Palmer MK, O’Leary $\mathrm{DH}$, et al. Effect of Rosuvastatin on Progression of Carotid Intima-Media Thickness in Low-Risk Individuals With Subclinical Atherosclerosis: The METEOR Trial. JAMA. 2007;297:1344-53.

5. Collins R, Peto R, Armitage J. The MRC/BHF Heart Protection Study: preliminary results. Int J Clin Pract. 2002;56:53-6.

6. Correti MC, Anderson TJ, Benjamin EJ, Celermajer D, Charbonneau F, et al. Guidelines for the ultrasound assessment of endothelial-dependent flow-mediated vasodilatation of the brachial artery: a report of the International Brachial Artery Reactivity Task Force. J Am Coll Cardiol. 2002;39:257-65.

7. Coimbra SR, Lage SH, Brandizi L, Yoshida V, da Luz PL. The action of red wine and purple grape juice on vascular reactivity is independent of plasma lipids in hypercholesterolemic patients. Braz J Med Biol Res. 2005;38:1339-47.

8. Benjo AM, Maranhão RC, Coimbra SR, Andrade AC, Favarato D, Molina MS, et al. Accumulation of chylomicron remnants and impaired vascular reactivity occur in subjects with isolated low HDL cholesterol: effects of niacin treatment. Atherosclerosis. 2006;187:116-22.

9. Redberg R, Vogel R, Criqui MH, Herrington DM, Lima JAC, Roman MJ. Task Force $\mathrm{N}^{\circ} 3$ - What Is Spectrum of Current and Emerging Techiniques for the nonivasive Measurement of Atherosclerosis? JACC. 2003:41;1856-97.

10. Stoffers HEJH, Kester ADM, Kaiser V, Rinkens PE, Kitslaar PJ, Knotttnerus JA. The diagnostic value of the measurement of the anklebrachial systolic pressure index in primary health care. J Clin Epidemiol. 1996;49:1401-5.

11. McKenna M, Wolfson S, Kuller L. The ratio of ankle and arm arterial pressure as an independent predictor of mortality. Atherosclerosis.1991;87:119-28.

12. Agatston AS, Janowitz WR, Hildner FJ, Zusmer NR, Viamonte M, Detrano R. Quantification of coronary artery calcium using ultra fast computed tomography. J Am Coll Cardiol. 1990;15:827-32.

13. Kronmal RA, McClelland RL, Detrano R, Shea S, Lima JA, Cushman M, et al. Risk Factors for the Progression of Coronary Artery Calcification in Symptomatic Subjects: Results From the Multi-Ethnic Study of Atherosclerosis (MESA). Circulation 2007;115;2722-30.

14. Rinqqvist I, Fisher LD, Mock M, Davis KB, Wedel H, Chaitman BR, et al. The Coronary Artery Surgery Study. Prognostic Value of Angiographic Indices of Coronary Artery Disease from the Coronary Artery Surgery Study (CASS), J Clin Invest. 1983;71:1854-66.

15. Escosteguy CC, Portela MC, Medronho RA, Vasconcelos MTL. Infarto agudo do miocárdio: perfil clínico-epidemiológico e fatores associados ao óbito hospitalar no município do Rio de Janeiro. Arq Bras Cardiol. 2003;80:593-9.

16. Castanho VS, Oliveira LS, Pinheiro HP, Oliveira HC, de Faria EC. Sex differences in risk factors for coronary heart disease: a study in a Brazilian population. BMC Public Health. 2001;1:3-41.
17. Martins IS, Gomes AD, Pasini U. [Lipemic levels and some cardiovascular disease risk factors in a population of the city of S. Paulo, Brazill. Rev. Saúde Publ., S. Paulo, 1989;23:26-38.

18. Wilson PW, D'Agostinho RB, Levy D, Belanger AM, Silbershatz H, Kannel WB. Prediction of coronary heart disease using risk factor categories. Circulation. 1998;97:1837-59.

19. Berenson GS, Srinivasan SR, Bao W, Newman WP 3rd, Tracy RE, Wattigney WA. Association between multiple cardiovascular risk factors and atherosclerosis in children and young adults. The Bogalusa Heart Study. N Engl J Med. 1998;338:1650-6.

20. The Lipid Research Clinics coronary Primary Prevention Trial. Results I: reduction in incidence of coronary heart disease. JAMA. 1984;251:35164.

21. Magalhães CC. [Low high density lipoprotein (HDL-c) cholesterol predicts cardiovascular mortality in clinical evolution in both gender after coronary artery bypass graft surgery ] São Paulo, 2002, Doctorate Thesis, Medicine School University of São Paulo.

22. Pepys MB, Hirschfield GM. C-reactive protein: a critical update. J Clin Invest. 2003;111:1805-12.

23. O`Keef JH, Cordain L, Jones P, Abuissa H. Coronary artery disease prognosis and C-reactive protein levels improve in proportion to percent lowering of low density lipoprotein. Am J Card. 2006;98:135-9.

24. Da Luz PL, Favarato D, Faria-Neto JR, Lemos P, Chagas AC. High ratio of triglycerides to HDL-cholesterol predicts extensive coronary disease. Clinics. 2008;63:427-32.

25. Da Luz PL, Cesena FHY, Favarato D, Cerqueira ES. Comparison of serum lipid values with coronary artery disease at 50,50 to 59,60 to 69 and $>70$ years of age. Am J Cardiol. 2005; 96:1640-3.

26. Taylor AJ, Feuerstein I, Wong H, Barko W, Brazaitis M, O'Malley PG. Do conventional risk factors predict subclinical coronary artery disease? Results form the prospective. Am Heart J. 2001;141:463-8.

27. Secci A, Wong N, Tang W, Wang S, Doherty T, Detrano R. Electron beamcomputed tomographic coronary calcium as a predictor of coronary events: comparison of two protocols. Circulation. 1997;96:1122-9.

28. Meneghelo RS, Santos RD, Almeida B, Hidal J, Martinez T, Moron $\mathrm{R}$, et al. Distribution of coronary artery calcium scores determined by ultrafast computed tomography in 2.253 asymptomatic white men. Arq Bras Cardiol. 2003;81(Suppl. VII):27-36.

29. Pletcher MJ, Tice JA, Pignone M, Browner WS. Using the coronary artery calcium score to predict coronary heart disease events: a systematic review and meta-analysis. Arch Intern Med. 2004;164:128592.

30. Greenland P, LaBree L, Azen SP, Doherty TM, Detrano RC. Coronary artery calcium score combined with Framingham score for risk prediction in asymptomatic individuals. JAMA. 2004;291:210-5.

31. O'Leary DH, Polak JF, Kronmal RA, Manolio TA, Burque GL, Wolfson SK Jr. Carotid-artery intima and media thickness as a risk factor for myocardial infarction and stroke in older adults: Cardiovascular Health Study Collaborative Research Group. N Engl J Med.1999;340:14-22.

32. Salonen JT, Salonen R. Ultrasound B-mode imaging in observational studies of atherosclerotic progression. Circulation.1993;87( Suppl II):56-65.

33. Kuvin JT, Karas RH. Clinical utility of endothelial function testing. Ready for prime time? Circulation. 2003;107:3243-7. 